\title{
Team-Based Clinic Redesign of Opioid Medication Man- agement in Primary Care: Effect on Opioid Prescribing
}

\author{
Michael L. Parchman, MD, MPH \\ Robert B. Penfold, $P b D^{1}$ \\ Brooke Ike, MPH ${ }^{2}$ \\ David Tauben, $M D^{3}$ \\ Michael Von Korff, $P b D^{1}$ \\ Mark Stephens, $B A^{4}$ \\ Kari A. Stephens, $P b D^{5}$ \\ Laura-Mae Baldwin, MD, MPH \\ 'Kaiser Permanente Washington Health \\ Research Institute, Seattle, Washington \\ ${ }^{2}$ Department of Family Medicine, Univer- \\ sity of Washington, Seattle, Washington \\ ${ }^{3}$ Department of Medicine, University of \\ Washington, Seattle, Washington
}

${ }^{4}$ Change Management Consulting, Seattle, Washington

${ }^{5}$ Department of Psychiatry and Behavioral Sciences, University of Washington, Seattle, Washington

Conflicts of interest: authors report none.

\section{CORRESPONDING AUTHOR}

Michael L. Parchman, MD, MPH Kaiser Permanente Washington Health Research Institute

1730 Minor Ave, Ste 1600

Seattle, WA 98101

michael.x.parchman@kp.org

\begin{abstract}
PURPOSE Six key elements of opioid medication management redesign in primary care have been previously identified. Here, we examine the effect of implementing these Six Building Blocks on opioid-prescribing practices.
\end{abstract}

METHODS Six rural-serving organizations with 20 clinic locations received support for 15 months during the period October 2015 to May 2017 to implement the Six Building Blocks. Patients undergoing long-term opioid therapy (LtOT) at these study sites were compared with patients undergoing LtOT enrolled in a regional health plan who did not receive care at the study sites but who resided in the same primary care service areas (control group). Outcomes were monthly trend in the proportion of patients undergoing LtOT prescribed a $\geq 100$ morphine equivalent dose (MED) of opioids daily and the total number of patients receiving an opioid prescription. An interrupted time series using difference-indifference analysis was used for tests of significance.

RESULTS The proportion of patients prescribed a $\geq 100$ MED of opioids daily decreased $2.2 \%(11.8 \%$ to $9.6 \%)$ among patients at the intervention clinics and $1.3 \%(14.0 \%$ to $12.7 \%)$ among patients in the control group. The rate of decrease was significantly greater among study patients than among patients in the control group $(P=.018)$. The rate of decrease in the number of patients on LtOT at intervention clinics increased during the intervention period compared with the preintervention period $(P<.001)$.

CONCLUSIONS Efforts to redesign opioid medication management in primary care resulted in a significant decrease in opioid prescribing. Future research is needed to determine if these results are generalizable to other settings and to assess implications for patient-reported outcomes.

Ann Fam Med 2019;17:319-325. https://doi.org/10.1370/afm.2390.

\section{INTRODUCTION}

U se of opioid medication long term for chronic pain has contributed to an epidemic of opioid overdose and addiction. ${ }^{1}$ Although rates of opioid prescribing have moderated in recent years, the amount of opioids prescribed in 2015 remained approximately 3 times that in $1999 .{ }^{2}$ In addition, there was a $59 \%$ increase in the use of long-term opioid therapy (LtOT), defined as use of opioids daily for chronic pain within a 3-month period, from 2006 to 2015. Overdose deaths involving prescription opioids were 5 times greater in 2016 than in 1999. ${ }^{3}$ Four of 5 heroin users began their addiction on prescription opioids, ${ }_{,}{ }^{4}$ and the total number of opioid overdose deaths annually has continued to increase each year through 2017. These trends are concerning, considering a growing body of evidence that LtOT is not effective for chronic pain. ${ }^{5}$

In response, clinical guidelines supporting more judicious use of opioids for chronic pain have been developed and disseminated. ${ }^{6}$ These guidelines are especially relevant to primary care, which prescribes the majority of opioids for chronic pain. ${ }^{7}$ Implementing recommendations embedded in clinical guidelines requires more than clinician awareness and education. ${ }^{8,9}$ Patient-centered care for chronic pain requires a team- 
based approach supported by changes to clinic systems and workflows within teams to ensure that care is safe and effective..$^{9-11}$

The opioid epidemic has had its most devastating effects in rural, disadvantaged populations. ${ }^{12}$ Although there have been efforts within larger integrated delivery systems to decrease opioid prescribing, ${ }^{13,14}$ it is unclear whether similar approaches can be used in smaller clinics serving disadvantaged populations because such clinics typically have fewer resources for quality improvement initiatives. In a prior study, we identified 6 common elements, the Six Building Blocks, for team-based redesign of opioid medication management within smaller primary care practice settings as follows: (1) provide leadership support, (2) revise and align clinic policies, use of patient agreements, and defined workflows, (3) track the patient population, (4) implement planned, patient-centered visits, (5) identify resources for complex patients, and (6) measure success. ${ }^{15}$ Here we report the results of a study implementing the Six Building Blocks at rural primary care clinics on (1) the proportion of patients on high-dose opioids and (2) the number of patients who continue to receive LtOT prescriptions across all clinics.

\section{METHODS}

\section{Study Setting and Subjects}

This study was reviewed and approved by the Institutional Review Board of the University of Washington. We conducted this study at the clinics of 6 ruralserving health care organizations. These 6 organizations provided care at 20 clinic locations across eastern Washington and central Idaho. Each clinic location had from 2.6 to 7.4 full-time equivalent clinicians. The organizations were members of the Washington, Wyoming, Alaska, Montana, and Idaho (WWAMI) region Practice Research Network, a primary care-practicebased research network in the 5-state WWAMI region. Five of the organizations, along with their associated ambulatory care clinics, were designated as critical access hospitals. Subjects were LtOT patients seen at the 20 clinics within these 6 rural-serving organizations that implemented the Six Building Blocks.

We used a quasi-experimental study design by identifying a comparison group of LtOT patients to serve as temporal control subjects for the patients who were exposed to the Six Building Blocks intervention at study sites. All LtOT patients in the comparison group were members of a large regional health plan and resided in the same primary care service areas as the clinics where the Six Building Blocks were implemented. ${ }^{16}$ The study sites did not accept patients on this health plan, so none of the patients in the compari- son group were exposed to the intervention. Information about the clinics where these patients received care was not available. This design uses this nonequivalent control group to control for secular trends in changes in opioid prescribing that are not attributable to the intervention. ${ }^{17}$

\section{Implementation Support for the Six Building Blocks Program}

Each organization received approximately 15 months of support, beginning with an in-person kickoff event during the period October 2015 to February 2016. During the study kick-off meeting, all members of the organization's clinics, including clinicians, nurses, medical assistants, and front desk staff, met with 3 members of the study team (M.L.P., L-M.B., B.I.). They were introduced to the Six Building Blocks, participated in a self-assessment activity regarding current opioid medication management clinic systems, and discussed their current approach to managing opioid medications for their patients with chronic pain and where they saw room for improvement. Each organization designated an opioid improvement team consisting of a clinic champion (a full-time clinician for each organization), a project manager (either a quality improvement professional or clinic manager), a data manager (generally a medical assistant), and others as desired by the clinic. One practice facilitator from the study team held quarterly individual check-in calls with each opioid improvement team leader to assist with their plans to implement elements of the Six Building Blocks. Opioid improvement team members also participated in monthly shared learning telephone calls with the other study sites, led by the practice facilitator. Clinicians at each clinic were invited to participate in optional twice-monthly TelePain webinars with a physician pain specialist at the University of Washington, which included didactic lectures and case presentations. Clinics and their opioid improvement teams had access to a study website with shared resources such as model clinic policies and patient agreements. Study organizations were also offered an online registry to track their patients on LtOT, but it did not officially launch until September 2016. Four sites used this support, but only 1 site used it consistently during the study. In addition, sites received reimbursement to cover the salary of a 0.1 full-time equivalent medical assistant to develop and enter data into their system to track LtOT patients.

\section{Data Collection}

For each study site, we requested a medication data extract from the electronic health records for all opioid prescriptions for patients aged 21 years or older start- 
Table 2. Trend in Proportion of Patients With a $\geq 100$ Morphine Equivalent Dose Daily

\begin{tabular}{lccccc}
\hline Variable & $d f$ & Coefficient & SE & $t$ Value & $P$ Value \\
\hline Interrupted time series, within study sites & & & \\
Intercept & 1 & 11.929 & 0.329 & 36.3 & $<.0001$ \\
Time (secular trend) & 1 & 0.358 & 0.351 & 1.02 & .320 \\
Change in level & 1 & -0.054 & 0.065 & -0.82 & .420 \\
Change in slope & 1 & -0.138 & 0.069 & -2.00 & .060 \\
Interrupted time series, intervention group vs control group & \\
Intercept & 1 & 3.943 & 0.448 & 8.8 & $<.0001$ \\
Time (secular trend) & 1 & -0.226 & 0.089 & -2.55 & .019 \\
Change in level & 1 & 1.592 & 0.478 & 3.33 & .003 \\
Change in slope & 1 & 0.242 & 0.094 & 2.58 & .018 \\
\hline
\end{tabular}

Table 3. Trend in Number of Patients on Long-term Opioid Therapy

\begin{tabular}{lccccc}
\hline Variable & df & Estimate & SE & $t$ Value & $P$ Value \\
\hline Interrupted time series, within study sites & & & \\
Intercept & 1 & 1,680 & 38.1 & 44.1 & $<.0001$ \\
Time (secular trend) & 1 & -133.0 & 40.7 & -3.27 & .004 \\
Change in level & 1 & 60.7 & 7.5 & 8.04 & $<.0001$ \\
Change in slope & 1 & -75.8 & 8.0 & -9.48 & $<.0001$ \\
\hline
\end{tabular}

$11.8 \%$ at the start of the intervention to $9.6 \%$ at 15 months. This reflects an average decrease of $0.15 \%$ per month across all study sites during the intervention. The $P$ value for the coefficient for the change in slope within study sites $(P=.06)$ indicates that the proportion of patients on high-dose opioids decreased faster during the study intervention period compared with the preintervention period. Over the same time, the proportion of patients in the control group who were on high-dose opioids decreased from $14.0 \%$ to $12.7 \%$, an average decrease of $0.09 \%$ per month. The rate of decrease in the proportion of patients on high-dose opioids was significantly greater in the study group compared with the control group $(P=.018)$. Comparing the rate of decrease between the study group and the control group, this translates to approximately 4 patients on high-dose opioids decreasing their daily dose to a $<100$ MED per month in the intervention group compared to 2.4 patients on high-dose opioids per month in the control group.

Table 2 shows the results of the interrupted time series regression of the proportion of patients on highdose opioids. In the intervention group, the proportion on high-dose opioids ( $\geq 100 \mathrm{MED}$ ) decreased from
Table 3 and Figure 1 show the results of the interrupted time series regression for the total number of patients meeting the LtOT definition. For the intervention group, this number decreased from 2,065 to

Figure 1. Number of LtOT patients by month.

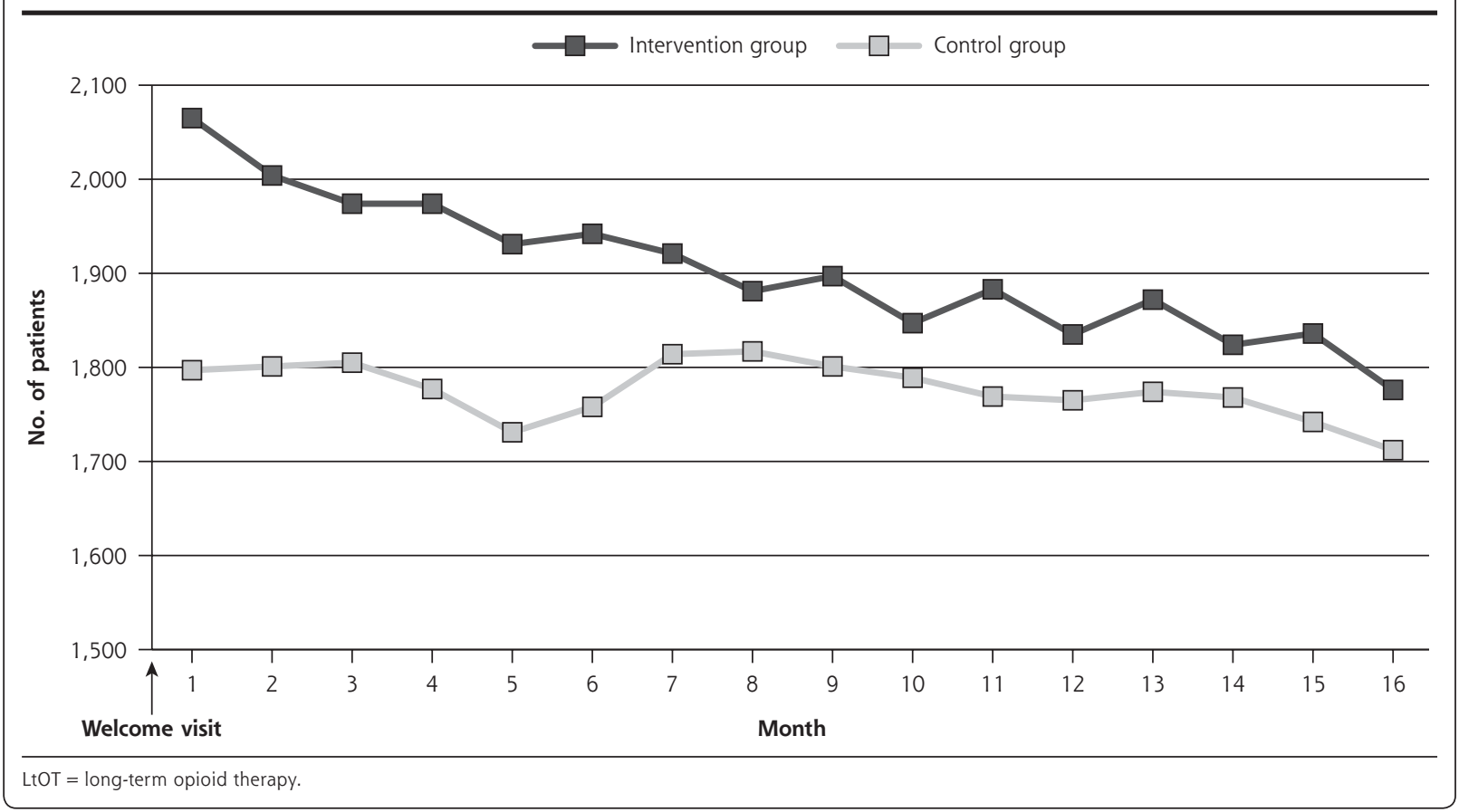


1,776 at the end of the 15 months of support, a $14.0 \%$ decrease. The coefficient for the change in slope $(-75.8)$ in Table 3 indicates that the rate of decrease in the total number of patients receiving an opioid prescription was greater during the intervention period compared with the preintervention period $(P<.001)$. The number of LtOT patients in the control group was 1,797 at the beginning of the intervention period and 1,712 at the end of the 15 months, a decrease of $4.7 \%$ (Figure 1). The difference-in-difference model comparing the intervention group to the control group for the trend in number of patients on LtOT violated the parallel trends assumption ${ }^{20}$ in the preintervention period, therefore, it is not presented.

We also examined the trend in MED categories across study sites over time (Figure 2). As the proportion of patients on high-dose opioids ( $\geq 100 \mathrm{MED}$ ) decreased over the study period, the proportion of patients in the 50-99 MED category remained relatively stable, whereas the proportion in the $<50 \mathrm{MED}$ category increased from approximately $75 \%$ to $78 \%$.

\section{DISCUSSION}

Clinic system redesign guided by the Six Building Blocks resulted in significant decreases in both the proportion of patients on high-dose opioids and the total number of patients receiving opioids in these rural health clinics. Although the within-study group interrupted time series analysis of the proportion on high-dose opioids did not reach statistical significance $(P=0.06)$, the decrease was greater than that seen in the comparison group of control patients from the same primary care service areas, suggesting that implementation of the Six Building Blocks accelerated an existing trend to prescribe opioids at lower doses. This finding is also supported by the decrease in the total number of patients receiving LtOT across the study sites and the shift in prescribed dose of opioids across the 3 categories of dose shown in Figure 2.

Although these decreases are modest, they reflect the initial phases of implementing new clinic systems for opioid medication management in the study settings. For example, we observed that revision and approval of new clinic policies across the sites required an average of 6 of the 15 months of support. In addition, differences in the sociodemographic characteristics of the intervention group versus the control group, especially the proportion of patients on public insurance, should have biased the results toward a lower effect size $e_{\text {; }}$ therefore, detecting a significant difference between the intervention and control groups is notable.

It is worth noting that none of the Six Building Blocks directly addresses tapering of opioids; therefore, the results are noteworthy in demonstrating that the systems-based changes instituted at the clinics had an indirect influence on clinicians' opioid-prescribing behaviors. How did implementation of the Six Build-

\section{Figure 2. Intervention clinic trend in proportion of patients by MED category.}

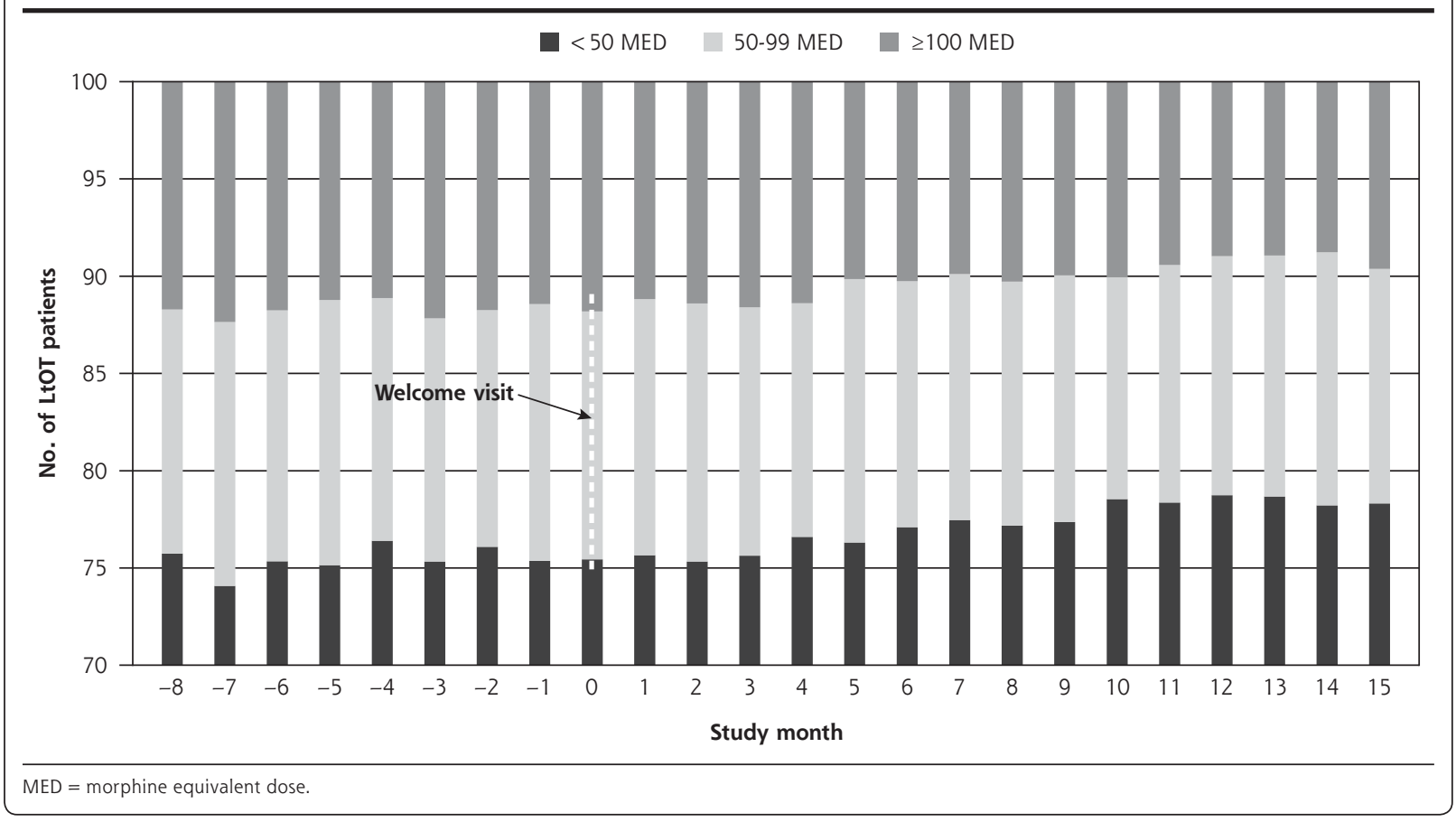


ing Blocks influence prescribing behaviors? It is possible that clinicians for both groups of patients, intervention and control, were aware of new opioidprescribing guidelines; thus the decreases seen in both intervention-site patients and the comparison control group. Enhanced awareness of the details of these guidelines and the evidence behind them, however, along with implementing new clinic systems to support guideline-recommended changes in care, may have led to more conversations with LtOT patients at the study sites compared with those in the comparison group about tapering their dose. Several clinicians at the study sites mentioned to study team members that some patients self-tapered their opioid dose in response to conversations about risks and harms. ${ }^{22}$ In addition, monitoring and reporting on prescribing behaviors by individual clinicians within these organizations created opportunities for discussions and peer comparisons that may have influenced subsequent prescribing behaviors. In a systematic review of dose reduction or discontinuation of LtOT, a common theme in studies with positive outcomes was team-based, multidisciplinary support of clinicians and staff. ${ }^{23}$

Earlier studies of efforts to change opioidprescribing patterns were conducted in large integrated delivery systems. ${ }^{13,14}$ An evaluation of the Group Health opioid initiative revealed a decrease in the prescribing of high-dose opioids ( $\geq 120 \mathrm{MED}$ ) from $16.8 \%$ to $6.3 \%$ over a period of 8 years. ${ }^{13}$ This compares to the decrease in the present study from $11.8 \%$ to $9.6 \%$ over a much shorter period of 15 months using a lower threshold of $\geq 100$ MED to define high dose. In a more recent trial of academic detailing, which used a registry and decision-support tools across 4 safety-net clinics, LtOT patients at intervention clinics were more likely to have a $\geq 10 \%$ decrease in MED. ${ }^{22}$ In a similar trial of academic detailing, which used audit and feedback as well as external facilitation, the average MED was decreased significantly at 12 months for intervention clinic patients compared with the comparison control group. ${ }^{24}$ These studies used blended implementation strategies similar to the ones used in the present study, suggesting that this approach can be effective in improving opioid-prescribing behaviors.

Of concern is the lack of data regarding the disposition of the 289 patients who were no longer receiving refills for their LtOT across the intervention study sites. These patients may have left the clinic to obtain refills elsewhere, replaced opioids with alternative treatments for their chronic pain, or of even greater concern, replaced prescription opioids with illicit drugs. This is important given that 4 of 5 heroin users start with prescription opioid misuse. ${ }^{4}$ It is critically important that future research track the disposition of patients who reduce or are no longer receiving opioid refills and measure patient-reported outcomes such as function and quality of life.

Further work is needed to study our approach at more diverse primary care sites to assess the effectiveness outside the 6 rural-serving health organizations we studied. We need to find ways to support rural clinics in redesigning care for their patients on LtOT and to tailor that support to their specific context. One possible approach is a partnered train-the-trainer model, similar to that tested at the Department of Veterans Affairs, in which an external expert trains and then provides support for an internal facilitator, such as a quality improvement lead, within each rural clinic organization..$^{25}$ It is also of critical importance to follow the effect of our program on patient disposition and patient-reported outcomes. Key questions are the clinical significance of the changes made and the disposition of patients who reduce or are no longer receiving opioid refills. Nonetheless, our data demonstrate that efforts to redesign care by primary care teams, guided by the Six Building Blocks framework, can improve opioid-prescribing practices to be more consistent with current guidelines.

To read or post commentaries in response to this article, see it online at http://www.AnnFamMed.org/content/17/4/319.

Key words: primary health care; analgesics; opioids; quality improvement; prescriptions

Submitted September 24, 2018; submitted, revised, February 22, 2019; accepted March 13, 2019.

Funding support: This study was supported by the Agency for Healthcare Research and Quality (AHRQ) (R18HS023750). The contents of this article are solely the responsibility of the authors and do not necessarily represent the official views of or imply endorsement by the AHRQ or the US Department of Health and Human Services. Additional support was provided by the National Center for Advancing Translational Sciences of the National Institutes of Health (UL1 TR002319). The content of this article is solely the responsibility of the authors and does not necessarily represent the official views of the National Institutes of Health.

Acknowledgments: We are grateful to the dedicated staff and clinicians of the primary care clinics across Washington and Idaho who are members of the WWAMI region Practice and Research Network and who bravely agreed to participate in this ambitious study.

\section{References}

1. Okie S. A flood of opioids, a rising tide of deaths. $N$ Engl J Med. 2010;363(21):1981-1985.

2. Guy GP Jr, Zhang K, Bohm MK, et al. Vital signs: changes in opioid prescribing in the United States, 2006-2015. MMWR Morb Mortal Wkly Rep. 2017;66(26):697-704.

3. Seth P, Rudd RA, Noonan RK, Haegerich TM. Quantifying the epidemic of prescription opioid overdose deaths. Am J Public Health. 2018;108(4):500-502. 
4. Jones CM. Heroin use and heroin use risk behaviors among nonmedical users of prescription opioid pain relievers - United States, 2002-2004 and 2008-2010. Drug Alcohol Depend. 2013;132(1-2): 95-100.

5. Krebs EE, Gravely A, Nugent S, et al. Effect of opioid vs nonopioid medications on pain-related function in patients with chronic back pain or hip or knee osteoarthritis pain: the SPACE Randomized Clinical Trial. JAMA. 2018;319(9):872-882.

6. Dowell D, Haegerich TM, Chou R. CDC guideline for prescribing opioids for chronic pain - United States, 2016. MMWR Recomm Rep. 2016;65(1):1-49.

7. Levy B, Paulozzi L, Mack KA, Jones CM. Trends in opioid analgesicprescribing rates by specialty, U.S., 2007-2012. Am J Prev Med. 2015;49(3):409-413.

8. Grol R. Successes and failures in the implementation of evidencebased guidelines for clinical practice. Med Care. 2001;39(8 Suppl 2): II46-II54.

9. Cabana MD, Rand CS, Powe NR, et al. Why don't physicians follow clinical practice guidelines? A framework for improvement. JAMA. 1999;282(15):1458-1465.

10. Steckowych K, Smith M. Workflow process mapping to characterize office-based primary care medication use and safety: a conceptual approach. Res Social Adm Pharm. 2018;S1551-S7411(17):30989-0.

11. Giannitrapani KF, Ahluwalia SC, McCaa M, Pisciotta M, Dobscha S, Lorenz KA. Barriers to using nonpharmacologic approaches and reducing opioid use in primary care. Pain Med. 2018;19(7): 1357-1364.

12. Case A, Deaton A. Rising morbidity and mortality in midlife among white non-Hispanic Americans in the 21st century. Proc Natl Acad Sci U S A. 2015;112(49):15078-15083.

13. Von Korff M, Dublin S, Walker RL, et al. The impact of opioid risk reduction initiatives on high-dose opioid prescribing for patients on chronic opioid therapy. J Pain. 2016;17(1):101-110.

14. Westanmo A, Marshall P, Jones E, Burns K, Krebs EE. Opioid dose reduction in a VA health care system-implementation of a primary care population-level initiative. Pain Med. 2015;16(5):1019-1026.
15. Parchman ML, Von Korff M, Baldwin LM, et al. Primary care clinic re-design for prescription opioid management. J Am Board Fam Med. 2017;30(1):44-51.

16. Goodman DC, Mick SS, Bott D, et al. Primary care service areas: a new tool for the evaluation of primary care services. Health Serv Res. 2003;38(1 Pt 1):287-309.

17. Shadish W, Cook T, Campbell D. Experimental and Quasi-Experimental Designs for Generalized Causal Inference. Boston, MA: Houghton Mifflin Company; 2002.

18. Penfold RB, Zhang F. Use of interrupted time series analysis in evaluating health care quality improvements. Acad Pediatr. 2013; 13(6 Suppl):S38-\$44.

19. Wagner AK, Soumerai SB, Zhang F, Ross-Degnan D. Segmented regression analysis of interrupted time series studies in medication use research. J Clin Pharm Ther. 2002;27(4):299-309.

20. Wing C, Simon K, Bello-Gomez RA. Designing difference in difference studies: best practices for public health policy research. Annu Rev Public Health. 2018;39:453-469.

21. Darnall BD, Ziadni MS, Stieg RL, Mackey IG, Kao MC, Flood P. Patient-centered prescription opioid tapering in community outpatients with chronic pain. JAMA Intern Med. 2018;178(5):707-708.

22. Liebschutz JM, Xuan Z, Shanahan CW, et al. Improving adherence to long-term opioid therapy guidelines to reduce opioid misuse in primary care: a cluster-randomized clinical trial. JAMA Intern Med. 2017;177(9):1265-1272.

23. Frank JW, Lovejoy TI, Becker WC, et al. Patient outcomes in dose reduction or discontinuation of long-term opioid therapy: a systematic review. Ann Intern Med. 2017;167(3):181-191.

24. Quanbeck A, Brown RT, Zgierska AE, et al. A randomized matchedpairs study of feasibility, acceptability, and effectiveness of systems consultation: a novel implementation strategy for adopting clinical guidelines for Opioid prescribing in primary care. Implement Sci. 2018;13(1):21.

25. Kirchner JE, Ritchie MJ, Pitcock JA, Parker LE, Curran GM, Fortney JC. Outcomes of a partnered facilitation strategy to implement primary care-mental health. J Gen Intern Med. 2014;29 Suppl 4: 904-912. 